\title{
-NOTES-
}

\section{NONLINEAR ANALYSIS OF A TWISTED AXIALLY LOADED ELASTIC ROD}

\author{
BY DA VID W. ZACHMANN (Colorado State University, Fort Collins)
}

\begin{abstract}
A slender, inextensible elastic rod is acted upon by a twisting couple and an axial load. The position of the rod's centerline is determined by two fourth-order, coupled, nonlinear boundary value problems, each of which contains two eigenparameters. These equilibrium equations admit the trivial solution for all values of the eigenparameters, i.e., for any axial load and any twisting couple. The linearized equilibrium equations have a countable number of eigencurves. Through using the implicit function theorem for Banach spaces it is shown that from each of the eigencurves of the linear problem there bifurcates a two-parameter sheet of nontrivial solutions of the nonlinear equilibrium equations.
\end{abstract}

1. Introduction. In this paper we consider a slender, inextensible, elastic rod with a circular cross-section. We choose our units so that the length of the rod can be taken to be one. In its undeformed state the position of the rod's centerline is given by

$$
(X, Y, Z)=(0,0, s), 0 \leq s \leq 1 .
$$

When the rod is subjected to the simultaneous action of an axial force $\mathbf{P}=P \mathbf{k}$ and a twisting couple $\mathbf{M}=M \mathbf{k}$, its centerline is located on some curve $\Gamma$ which can be written in parametric form

$$
\Gamma:(X, Y, Z)=(x(s), y(s), s+\zeta(s)), \quad 0 \leq s \leq 1 .
$$

To insure that the twisting couple is conservative $[1,5]$ we impose the following boundary conditions:

$$
\begin{aligned}
& x(0)=y(0)=x^{\prime}(0)=y^{\prime}(0), \\
& x(1)=y(1)=x^{\prime}(1)=y^{\prime}(1)=0, \\
& \zeta(0)=0 .
\end{aligned}
$$

These boundary conditions require that one end of the rod is fixed at the origin; the other end is restricted to lie on the $Z$-axis and the unit vector tangent to the rod's centerline is $\mathrm{k}$ at both ends.

It is shown in Appendix A that $x(s)$ and $y(s)$ satisfy

$$
\begin{aligned}
& {\left[\left(x^{\prime \prime}+2 \lambda y^{\prime}\right)\left(1-x^{\prime 2}-y^{\prime 2}\right)^{-1 / 2}+\mu x\right]^{\prime \prime}=0,} \\
& {\left[\left(y^{\prime \prime}-2 \lambda x^{\prime}\right)\left(1-x^{\prime 2}-y^{\prime 2}\right)^{-1 / 2}+\mu y\right]^{\prime \prime}=0}
\end{aligned}
$$

where $2 \lambda=M(E I)^{-1}, \mu=P(E I)^{-1}$ and $E I$ is the flexural rigidity of the rod. If (1.1) has been solved, then, as shown in Appendix A, $\zeta(s)$ can be recovered from

$$
\zeta^{\prime}(s)=-1+\left(1-x^{\prime 2}-y^{\prime 2}\right)^{1 / 2}, \zeta(0)=0 .
$$

Thus in order completely to solve the problem it is sufficient to consider (1.1) subject to $(\mathrm{BC} 1)$ and $(\mathrm{BC} 2)$. 
If $x \equiv y \equiv 0$ we say the rod is in an unbuckled state. A ny nontrivial solution of (1.1) satisfying $(\mathrm{BC} 1)$ and $(\mathrm{BC} 2)$ will be referred to as a buckled state of the rod. It is easy to see that the unbuckled state is mathematically possible for all values of the parameters $\lambda$ and $\mu$. In what follows we show that if $\left(\lambda_{0}, \mu_{0}\right)$ satisfies a certain transcendental equation, then $\left(\lambda_{0}, \mu_{0}\right)$ is critical in the sense that in every neighborhood there are values of $\lambda$ and $\mu$ which yield a buckled state of the rod.

2. The linear problem. Let $B$ be the complex Banach space consisting of all four times continuously differentiable complex-valued functions $z(s)=x(s)+i y(s), 0 \leq s \leq 1$, satisfying $z(0)=z^{\prime}(0)=0=z(1)=z^{\prime}(1)$. If $z(s) \in B$, the norm of $z$ is defined by

$$
\|z(s)\|=\sum_{j=0}^{4} \max _{0 \leq s \leq 1}\left|z^{(j)}(s)\right| .
$$

If we define $G(z, \lambda, \mu)$ by

$$
G(z, \lambda, \mu)=\left[\left(z^{\prime \prime}-2 i \lambda z^{\prime}\right)\left(1-\left|z^{\prime}\right|^{2}\right)^{-1 / 2}+\mu z\right]^{\prime \prime}
$$

then the system of equations (1.1) subject to $(\mathrm{BC} 1)$ and $(\mathrm{BC} 2)$ is equivalent to

$$
G(z, \lambda, \mu)=0, \quad z \in B .
$$

Since $z \equiv 0$ satisfies $(2.1)$ for all $(\lambda, \mu)$, it is appropriate to view $(2.1)$ as a nonlinear eigenvalue problem with $\lambda$ and $\mu$ representing two independent eigenparameters. In order to locate the values of $\lambda$ and $\mu$ at which a buckled state of the rod might appear, we turn to the Frechét derivative at zero of (2.1). An easy calculation shows this Frechét derivative to be

$$
L(z, \lambda, \mu)=0, \quad z \in B
$$

where

$$
L(z, \lambda, \mu)=\left[z^{\prime \prime}-2 i \lambda z^{\prime}+\mu z\right]^{\prime \prime} .
$$

If $\mu \neq 0$ then $L(z, \lambda, \mu)=0$ implies

$$
z(s)=a+b s+c \exp (i(\lambda+k) s)+d \exp (i(\lambda-k)), k=\left(\lambda^{2}+\mu\right)^{1 / 2}
$$

where $a, b, c$ and $d$ are complex constants. If the function (2.4) is to be in $B$, then $a-d$ must satisfy a system of four homogeneous linear equations determined by $z(0)=z^{\prime}(0)=0=$ $z(1)=z^{\prime}(1)$. Some computation shows that, if $\mu \neq 0, L(z, \lambda, \mu)=0$ has a nontrivial solution if and only if

$$
\mu \sin k+2 k(\cos k-\cos \lambda)=0, \quad k=\left(\lambda^{2}+\mu\right)^{1 / 2} .
$$

Eq. (2.5) agrees with the results of Beck [1] and Trosch [4].

If there is no axial thrust on the rod, $\mu=0$ and we see that $L(z, \lambda, 0)=0$ implies

$$
z(s)=a+b s+c s^{2}+d \exp (2 i \lambda s) .
$$

Again if the function (2.6) is in $B$ then $a-d$ must satisfy a homogeneous system of linear equations which can be shown to have a nontrivial solution if and only if

$$
\lambda=\tan \lambda \text {. }
$$

We will say that any point $(\lambda, \mu), \mu \neq 0$, satisfying (2.5) or any point $(\lambda, 0), \lambda \neq 0$, satisfying (2.7) is an eigenpoint of the linear operator $L$. 
The computations leading to (2.5) and (2.7) show that the coefficient matrix for the homogeneous system in $a-d$ always has rank of at least three. This implies that all of the eigenpoints are geometrically simple in the sense that if $L\left(z^{*}, \lambda^{*}, \mu^{*}\right)=0$ with $z^{*} \neq 0$, then $z^{*}$ is unique up to a multiplicative constant.

If the rod is subjected to only an axial thrust, $\lambda=0$, then (2.5) reduces to

$$
\mu \sin \mu^{1 / 2}+2 \mu^{1 / 2}\left(\cos \mu^{1 / 2}-1\right)=0 .
$$

Since (2.5) applies only for $\mu \neq 0$ we see, using the half-angle formulas, that (2.8) is equivalent to

$$
\left(\mu^{1 / 2} \cos \left(\frac{1}{2} \mu^{1 / 2}\right)-2 \sin \left(\frac{1}{2} \mu^{1 / 2}\right)\right) \sin \frac{1}{2} \mu^{1 / 2}=0
$$

which shows that either

$$
\mu=(2 n \pi)^{2}, \quad n=1,2, \cdots
$$

or

$$
\frac{1}{\sqrt{2}} \sqrt{\mu}=\tan \left(\frac{1}{2} / \mu\right) .
$$

Let $\mu_{2 k-1}=(2 k \pi)^{2}, k=1,2, \cdots$ and let $\mu_{2 k}, k=1,2, \cdots$ denote the $k$ th positive solution of (2.11). Note that

$$
\mu_{2 k-1}<\mu_{2 k}<\mu_{2 k+1}, \quad k=1,2, \cdots .
$$

This shows that the eigenpoints on the $\mu$-axis are of the form $\left(0, \mu_{n}\right), n=1,2, \cdots$, with the $\mu_{n}$ as defined above. Similarly, the eigenpoints on the $\lambda$-axis are given by $\left( \pm \lambda_{n}, 0\right)$ where $\lambda_{n}$ denotes the $n$th positive root of (2.7). A numerical analysis of Eq. (2.5) shows that there exists a countable sequence of continuous curves of the form

$$
\mu=g_{n}(\lambda), \quad n=1,2, \cdots
$$

and that $(\lambda, \mu)=\left(\lambda, g_{n}(\lambda)\right)$ satisfies (2.5). Moreover, $g_{n}(0)=\mu_{n}$, and $g_{n}(\lambda)=0$ implies $\lambda=$ $\pm \lambda_{n}$. Since each point of the form $(\lambda, \mu)=\left(\lambda, g_{n}(\lambda)\right)$ is an eigenpoint of $L$, we will refer to the curves (2.12) as eigencurves of $L$.

In summary, the linear operator $L$ possesses a countable sequence of eigencurves and at each eigenpoint $(\lambda, \mu)=\left(\lambda, g_{n}(\lambda)\right) L z=0$ has a nontrivial solution $z_{n}(s ; \lambda)$ which is unique up to a multiplicative constant. In closing this section we point out that computations show the curves (2.12) resemble parabolas opening downward.

3. Branching analysis. We call $\left(\lambda_{0}, \mu_{0}\right)$ a bifurcation point of the equation $G(z, \lambda, \mu)$ $=0, z \in B$, if for arbitrary $\rho>0$ the region

$$
\left|\lambda-\lambda_{0}\right|+\left|\mu-\mu_{0}\right|+\|z\|<\rho
$$

contains a solution $\left(z^{*}, \lambda^{*}, \mu^{*}\right), z^{*} \not \equiv 0$, of Eq. (2.1). If each point on some curve $C$ in the $\lambda-\mu$ plane is a bifurcation point of (2.1) we say $C$ is a bifurcation curve of $(2.1)$.

In this section we show that each of the eigencurves $\mu=g_{n}(\lambda)$ of the linearized problem (2.2) is a bifurcation curve of (2.1). We carry out the proof in detail for only the principal eigencurve, $C_{1}=\left\{(\lambda, \mu): \mu=g_{1}(\lambda)\right\}$, since the same methods apply to the other eigencurves. Using an implicit function theorem argument we show that bifurcating from $C_{1}$ is a two-parameter family of solutions $z=z(s ; \lambda, \epsilon)$ of $(2.1)$. This two-parameter family of

\footnotetext{
* Received August 9, 1977.
} 
solutions might be thought of as a sheet of solutions branching from $C_{1}$ in contrast to the curve of solutions which often bifurcates from an eigenvalue in one-parameter bifurcation problems.

It is convenient to view the elements of $B$ as elements of an inner product space with the inner product $\langle u, v\rangle$ defined by

$$
\langle u, v\rangle=\int_{0}^{1} \overline{u v} d s, \quad u, v \in B .
$$

Recall that the linear problem

$$
L\left(z, \lambda, g_{1}(\lambda)\right)=0, \quad z \in B
$$

has for each $\lambda$ a nontrivial solution $z(s, \lambda)$ which is unique up to a multiplicative constant. Let $z_{1}=z_{1}(s, \lambda)$ denote a solution of (3.2) which has been normalized subject to the condition $\left\langle z_{1}, z_{1}\right\rangle=1$.

Using $z_{1}$ and (3.1), we can define projection operators $P$ and $Q$ as follows:

$$
\begin{aligned}
& P z=\left\langle z, z_{1}\right\rangle z_{1}, \\
& Q z=z-P z .
\end{aligned}
$$

Note that (3.3) and (3.4) define projections on the space $C^{0}$ which consists of all continuous complex-valued functions of $s, 0 \leq s \leq 1$. As a final preliminary to our bifurcation analysis we define Banach spaces $B_{1}$ and $B_{2}$, each with the appropriate uniform norm, by

$$
B_{1}=\{z(s): z \in B, P z=0\}, B_{2}=\left\{z(s): z \in C^{0}, P z=0\right\} .
$$

If we define $N(z, \lambda)$ by

$$
N(z, \lambda)=G(z, \lambda, \mu)-L(z, \lambda, \mu)
$$

we see that

$$
N(z, \lambda)=\left\{\left[z^{\prime \prime}-2 i \lambda z^{\prime}-\left(z^{\prime \prime}-2 i \lambda z^{\prime}\right)\left(1-\left|z^{\prime}\right|^{2}\right)^{1 / 2}\right]\left(1-\left|z^{\prime}\right|^{2}\right)^{-1 / 2}\right\}^{\prime \prime} .
$$

Eq. (2.1) can be reformulated as

$$
L(z, \lambda, \mu)+N(z, \lambda)=0, \quad z \in B .
$$

We will look for a two-parameter family of solutions of (3.6) in the form

$$
(z, \lambda, \mu)=(z(s, \lambda, \epsilon), \lambda, \mu(\lambda, \epsilon))
$$

where

$$
\begin{aligned}
\mu(\lambda, \epsilon) & =g_{1}(\lambda)+\eta(\lambda, \epsilon), \quad \eta(\lambda, 0)=0, \\
z(s ; \lambda, \epsilon) & =\epsilon\left(z_{1}(s ; \lambda)+Z(s ; \lambda, \epsilon)\right), \quad Z(s ; \lambda, 0)=0,
\end{aligned}
$$

and $Z(s ; \lambda, \epsilon) \in B_{1}$.

If we substitute (3.7) into (3.6) and use the fact that $L\left(z_{1}, \lambda, g_{1}(\lambda)\right)=0$ we have

$$
L\left(Z, \lambda, g_{1}(\lambda)\right)+\eta z_{1}+\eta Z+\epsilon^{-1} N\left(\epsilon\left(z_{1}+Z\right), \lambda\right)=0 \text {. }
$$

An integration by parts shows that

$$
\left\langle L\left(Z, \lambda, g_{1}(\lambda)\right), z_{1}\right\rangle=\left\langle Z, L\left(z_{1}, \lambda, g_{1}(\lambda)\right\rangle .\right.
$$

Therefore, if we apply the projection $P$ to (3.8) and keep in mind that $z_{1}$ satisfies (3.2) we 
obtain

$$
\eta+\left\langle\epsilon^{-1} N\left(\epsilon\left(z_{1}+Z\right), \lambda\right), z_{1}\right\rangle=0 .
$$

Applying the projection $Q$ to (3.8) yields

$$
L\left(Z, \lambda, g_{1}(\lambda)\right)+\eta Z+Q\left(\epsilon^{-1} N\left(\epsilon\left(z_{1}+Z\right), \lambda\right)\right)=0 .
$$

Since $u \in C^{0}$ satisfies $u \equiv 0$ if and only if $P u=0=Q u$, it follows that (3.8) is equivalent to the pair of equations (3.9) and (3.10).

Let $F_{1}(Z, \eta ; \lambda, \epsilon)$ denote the left side of Eq. (3.10) and $F_{2}(Z, \eta ; \lambda, \epsilon)$ denote the left side of (3.9). A mapping $F$ from $B_{1} \times \mathbb{R}^{3}$ into $B_{2} \times R$ can be defined by

$$
F(Z, \eta ; \lambda, \epsilon)=\left(F_{1}(Z, \eta ; \lambda, \epsilon), F_{2}(Z, \eta ; \lambda, \epsilon)\right) .
$$

If we expand the term $\left(1-\left|z^{\prime}\right|^{2}\right)^{1 / 2}$ using the binomial theorem it is easy to see that

$$
\lim _{\epsilon \rightarrow 0} \epsilon^{-1} N\left(\epsilon\left(z_{1}+Z\right), \lambda\right)=\lim _{\epsilon \rightarrow 0} \epsilon^{-1} N_{Z}\left(\epsilon\left(z_{1}+Z\right), \lambda\right)=0 .
$$

From (3.11) it follows that the Frechét derivative of $F$ with respect to $Z$ and $\eta$ at $(Z, \eta)=$ $(0,0), \epsilon=0$, is given by

$$
\left.\frac{\partial\left(F_{1}, F_{2}\right)}{\partial(Z, \eta)}\right|_{\epsilon=0}=\left[\begin{array}{cc}
L\left(\cdot, \lambda, g_{1}(\lambda)\right) & 0 \\
0 & 1
\end{array}\right]
$$

Since (3.12) is an invertible transformation from $B_{1} \times R^{3}$ to $B_{2} \times R$ it follows from the implicit function theorem [3] that, for fixed $\lambda=\lambda_{0}$, there exists a local one-parameter family of solutions $Z(s ; \lambda, \epsilon), \eta(\lambda, \epsilon)$ of (3.9)-(3.10). Moreover, since $F_{1}$ and $F_{2}$ are continuous in $\lambda$ we have the following.

THEOREM. If $|\epsilon|$ is sufficiently small, there exists a two-parameter family of solutions ( $z(s$; $\lambda, \epsilon), \lambda, \mu(\lambda, \epsilon)$ ) of (2.1). $z$ and $\mu$ are continuous in $\lambda$ and $\epsilon$ and

$$
\lim _{\epsilon \rightarrow 0} \mu(\lambda, \epsilon)=g_{1}(\lambda), \lim _{\epsilon \rightarrow 0} z(s ; \lambda, \epsilon)=0 .
$$

COROLLARY. If $|\epsilon|$ is sufficiently small, there is a two-parameter sheet of buckled solutions $(x(s ; \lambda, \epsilon), y(s ; \lambda, \epsilon), \lambda, \mu(\lambda, \epsilon))$ satisfying (1.1), (BC1) and (BC2) which bifurcates from the eigencurve $\mu=g_{1}(\lambda)$.

Appendix A. Let $\mathrm{t}=\left(x^{\prime}(s), y^{\prime}(s), 1+\zeta^{\prime}(s)\right)$ denote a unit tangent to the curve $\Gamma$. In [2, pp. 79-81] it is shown that the bending moment $\mathbf{B}$ acting on a rod of circular crosssection is given by

$$
\mathbf{B}=E I\left(\mathbf{t} \times \mathbf{t}^{\prime}\right)+M \mathbf{t} .
$$

Also, since the external force $-P \mathbf{k}$ and the moment $\mathbf{M}=M \mathbf{k}$ are applied at the ends of the rod, it follows, from [2], that the equilibrium equations for the rod are given by

$$
\mathbf{B}^{\prime \prime}+P \mathbf{k} \times \mathbf{t}^{\prime}=0
$$

where $\mathbf{k}$ is a unit vector in the $Z$-direction. Writing (A2) in component form yields

$$
\begin{gathered}
{\left[E I\left(y^{\prime} \zeta^{\prime \prime}-y^{\prime \prime}\left(1+\zeta^{\prime}\right)\right)+M x^{\prime}-P y\right]^{\prime \prime}=0,} \\
{\left[E I\left(x^{\prime \prime}\left(1+\zeta^{\prime}\right)-x^{\prime} \zeta^{\prime \prime}\right)+M y^{\prime}+P x\right]^{\prime \prime}=0,} \\
{\left[E I\left(x^{\prime} y^{\prime \prime}-x^{\prime \prime} y^{\prime}\right)+M\left(1+\zeta^{\prime}\right)\right]^{\prime \prime}=0 .}
\end{gathered}
$$


The boundary conditions on $x$ and $y$ are that they together with their first derivatives vanish at $s=0$ and $s=1$. This implies $\zeta^{\prime}(0)=0=\zeta^{\prime}(1)$. By using these boundary conditions (A5) can be integrated twice to obtain

$$
E I\left(x^{\prime} y^{\prime \prime}-x^{\prime \prime} y^{\prime}\right)+M\left(1+\zeta^{\prime}\right)=M .
$$

Since $|\mathbf{t}|=1$,

$$
1+\zeta^{\prime}=\left(1-x^{\prime 2}-y^{\prime 2}\right)^{1 / 2}
$$

from which it follows that $\zeta^{\prime \prime}$ is given by

$$
\zeta^{\prime \prime}=-\left(x^{\prime} x^{\prime \prime}+y^{\prime} y^{\prime \prime}\right)\left(1+\zeta^{\prime}\right)^{-1} .
$$

Using (A7) and (A8) we eliminate $\zeta^{\prime}$ and $\zeta^{\prime \prime}$ from (A3) as follows:

$$
\begin{aligned}
\left(\left(y^{\prime} \zeta^{\prime \prime}-y^{\prime \prime}\left(1+\zeta^{\prime}\right)\right)\right. & =\left(-\left(y^{\prime} x^{\prime} x^{\prime \prime}+y^{\prime 2} y^{\prime \prime}\right)-y^{\prime \prime}\left(1+\zeta^{\prime}\right)^{2}\right)\left(1+\zeta^{\prime}\right)^{-1} \\
& =\left(x^{\prime}\left(x^{\prime} y^{\prime \prime}-x^{\prime \prime} y^{\prime}\right)-y^{\prime \prime}\right)\left(1+\zeta^{\prime}\right)^{-1} \\
& =\left(2 \lambda x^{\prime}-y^{\prime \prime}\right)\left(1-x^{\prime 2}-y^{\prime 2}\right)^{-1 / 2}-2 \lambda x^{\prime} .
\end{aligned}
$$

The last equality follows from (A6) and the fact that $2 \lambda=M(E I)^{-1}$. If we substitute the above into (A3) and recall that $\mu=P(E I)^{-1}$ we have

$$
\left[\left(y^{\prime \prime}-2 \lambda x^{\prime}\right)\left(1-x^{\prime 2}-y^{\prime 2}\right)^{-1 / 2}+\mu y\right]^{\prime \prime}=0
$$

which is the second of Eqs. (1.1). The first equation in (1.1) is obtained from (A4) in exactly the same way.

\section{REFERENCES}

[1] M. Beck, Knickung gerader Stäbe durch Druck und konservative Torsion. Ing.-Arch. 23, $231-253$ (1955)

[2] L. D. Landau and E. M. Lifshitz, Theory of elasticity, Addison-Wesley, Reading, Mass., 1959

[3] D. H. Sattinger, Topics in stability and bifurcation theory, Springer-Verlag Lecture Notes, No. 309,1973

[4] A. Trosch, Ing.-A rch. 20, 265 (1952)

[5] H. Ziegler, Principles of structural stability, Blaisdell, New York, 1968 